\title{
Impacto da incorporação de escuma em lodo de esgoto com fins agrícolas
}

\section{Impact of the scum incorporation in sewage sludge with agricultural purposes}

\author{
Data de entrada: \\ $11 / 02 / 2015$ \\ Data de aprovação: \\ 01/12/2015
}

\author{
Bárbara Zanicotti Leite Ross / Charles Carneiro / Clodoaldo José Marques / Fernanda Janaína Oliveira Gomes \\ da Costa / Sandro Froehner / Miguel Mansur Aisse
}

DOI 10.4322/dae.2016.002

\section{Resumo}

Foram desaguados separadamente em leitos de secagem na ETE Vassoural, Guarapuava, PR, 5 lotes de escuma e de lodo, produzidos em reator tipo UASB tratando esgotos domésticos. Após o desaguamento foram realizadas 3 misturas nas proporções 10, 20 e 50 \% em massa de escuma em lodo. Os parâmetros avaliados foram: sólidos totais e voláteis, óleos e graxas, coliformes termotolerantes, Salmonella spp., ovos viáveis de helmintos, $\mathrm{N}$ (Kjedahl, $\mathrm{NH}_{4}+$, $\mathrm{NO}_{2}-$ e $\mathrm{NO}_{3}-3$ ), $\mathrm{C}$ orgânico, a concentração total dos elementos $\mathrm{P}, \mathrm{K}, \mathrm{Na}, \mathrm{S}, \mathrm{Ca}$, $\mathrm{Mg}, \mathrm{Ar}, \mathrm{Ba}, \mathrm{Cd}, \mathrm{Pb}, \mathrm{Cu}, \mathrm{Cr}$, Hg, Mo, Ni, Se e Zn. Os tratamentos não apresentaram variância significativa $\left(\mathrm{P}_{0,05}\right)$ para nenhum dos parâmetros, indicando que a incorporação de escuma nas proporções de 10, 20 e $50 \%$ em massa não alterou a composição do lodo de esgoto destinado ao uso agrícola.

Palavras-chave: escuma, lodo anaeróbio de esgoto, reator UASB.

\section{Abstract}

Five treatments of municipal dewatered scum and sewage sludge from a UASB reactor were tested in drying beds, in terms of proportion of each one into the blend, considering agricultural soils as disposal. The treatments were the addition of 10, 20 and 50\% of scum mass in the mix scum/sewage sludge. The parameters monitored are: volatile and total solids; oils and greases; fecal coliforms; Salmonella spp; helminthes viable eggs; NKjedahl, $\mathrm{N}-\mathrm{NH}_{4}+, \mathrm{N}^{-\mathrm{NO}_{2}-\text {, }}$ $\mathrm{N}-\mathrm{NO}_{3}-3$; C organic; total concentration of $\mathrm{P}, \mathrm{K}, \mathrm{Na}, \mathrm{S}, \mathrm{Ca}, \mathrm{Mg}, \mathrm{Ar}, \mathrm{Ba}, \mathrm{Cd}, \mathrm{Pb}, \mathrm{Cu}, \mathrm{Cr}, \mathrm{Hg}, \mathrm{Mo}, \mathrm{Ni}$, Se and $\mathrm{Zn}$. Significant statistical difference $\left(P_{0,05}\right)$ were not observed for all parameters, which suggests that the scum addition from 10 up to $50 \%$ in the blend does not promote variation in the sewage sludge composition.

Keywords: scum, sewage sludge, UASB reactor.

\footnotetext{
Bárbara Zanicotti Leite Ross ${ }^{1}$ - Pesquisadora da Companhia de Saneamento do Paraná (Sanepar). Engenheira de Alimentos pela Pontifícia Universidade Católica do Paraná (PUCPR). Mestre em Tecnologia Química pela Universidade Federal do Paraná (UFPR). Doutora no Programa de Pós-Graduação em Engenharia de Recursos Hídricos e Ambiental da UFPR (2015).

Charles Carneiro ${ }^{1}$ - Gerente de Pesquisa e Desenvolvimento da Sanepar. Professor do Mestrado em Governança e Sustentabilidade do Instituto Superior de Administração e Economia da Fundação Getulio Vargas (ISAE/FGV) e da Pós-Graduação em Economia e Meio Ambiente da UFPR. Pós-Doutor em Engenharia e Ciência da Água pelo Instituto de Educação para as Águas (UNESCO-IHE).

Clodoaldo José Marques ${ }^{1}$ - Técnico em Química. Gestor de estação de tratamento de esgotos da Sanepar.

Fernanda Janaína Oliveira Gomes da Costa ${ }^{1}$ - Pesquisadora da Sanepar. Engenheira de Alimentos pela Universidade Estadual de Ponta Grossa (UEPG). Mestre em Ciência e Tecnologia de Alimentos pela mesma universidade. Doutora em Engenharia de Alimentos pela UFPR.

Sandro Froehner ${ }^{2}$ - Professor adjunto do Programa de Pós-Graduação em Engenharia de Recursos Hídricos e Ambiental (PPGERHA) e do curso de Engenharia Ambiental da UFPR.

Miguel Mansur Aisse ${ }^{2}$ - Professor associado do PPGERHA e do curso de Engenharia Civil da UFPR. Doutor em Engenharia Civil pela Escola Politécnica da Universidade de São Paulo (Poli-USP).

"Endereço para correspondência: ${ }^{1}$ Rua Engenheiro Antônio Batista Ribas, 151 - Tarumã - Curitiba-PR - CEP 82800-130. E-mail do autor principal: barbarazlasanepar.com.br. | ${ }^{2}$ Programa de Pós-Graduação em Engenharia de Recursos Hídricos e Ambiental - Universidade Federal do Paraná - Departamento de Hidráulica e Saneamento. Centro Politécnico - Jardim das Américas - Curitiba-PR - CEP 81531-990.
} 


\section{INTRODUÇÃO}

As três principais tecnologias utilizadas em países da América Latina e Caribe para o tratamento de esgotos domésticos são lagoas de estabilização, lodos ativados e reator anaeróbico de fluxo ascendente em manto de lodo (UASB), que possuem diferenças significativas na concepção, design e padrões de eficiência. Segundo Chernicharo (2004), o reator UASB é a alternativa mais empregada nos novos projetos de estações de tratamento no Brasil, porém necessita de pós-tratamento para que o efluente atinja os padrões preconizados pela legislação e de melhorias para diminuir problemas operacionais relacionados à retirada de escuma (SOUZA, 2006). Ocasionalmente, o acúmulo de escuma gera problemas hidráulicos e operacionais, além de diminuição na eficiência do tratamento. Seu manejo no interior dos reatores não é uma operação fácil e a destinação do resíduo é outro fator dificultador, especialmente em função da variabilidade na composição e do alto teor de umidade.

A escuma é constituída geralmente por materiais flotáveis e não degradados, notadamente Óleos e Graxas (O\&G), ceras, sabões, lodo e material particulado de forma geral (SILVA et al., 2005; SOUZA et al., 2006), muito embora a quantidade e características dependam da composição do substrato e do sistema preliminar de tratamento (LOBATO et al., 2007). Esses materiais, ao flotar na superfície dos reatores UASB e na ausência de um dispositivo que impeça sua saída com o esgoto tratado, acumulam-se, formando a escuma. Cabe destacar a diferença entre escuma e espuma: apesar de ambas serem menos densas que a água, flutuarem na superfície de reatores e apresentarem O\&G, bolhas de gás aderidas e micro-organismos, os processos de formação têm origens distintas, de modo que o controle é particularizado (SOUZA, 2006). Normalmente, a espuma ocorre em tanques de aeração quando as taxas de inclusão de ar são elevadas; além disso, a concentração de sólidos em suspensão, a relação alimento/microrganismo (F/M) e a baixa vazão de entrada são fatores que levam à formação de espuma em tanques de lodos ativados (WEF; ASCE; EWRI, 2005). Quanto mais eficiente for o tratamento preliminar, menos escuma será formada e melhor será a qualidade dessa composição.

Nos reatores UASB, o acúmulo de escuma ocorre em dois compartimentos distintos: na superfície da área de decantação e no interior do separador trifásico, os quais apresentam diferenças funcionais importantes na formação da escuma. No interior do separador, há um importante desprendimento de biogás, enquanto na superfície do decantador não deve haver esse desprendimento. A liberação de gases no interior do separador poderia ter um efeito controlador sobre a espessura e consistência da camada de escuma, devido à turbulência que causa na superfície hídrica (SOUZA et al., 2006).

De acordo com Souza et al. (2006), a formação de escuma é intensificada quando os reatores UASB operam com baixos tempos de detenção; isso porque a alta velocidade ascensional aumenta a quantidade de sólidos arrastados para a superfície do reator, contribuindo para uma maior formação de escuma. Estudo com três reatores UASB-piloto mostrou que, com tempo de detenção hidráulica menor $(5 \mathrm{~h})$ e maior velocidade ascensional $(1,1$ $\mathrm{m} / \mathrm{h}$ ), ocorreu maior arraste e/ou flotação da biomassa dos reatores. Outro parâmetro de operação dos reatores UASB que pode influenciar a formação da escuma é o descarte de lodo. A não retirada do excedente com frequência adequada provoca uma maior perda de sólidos para o compartimento de decantação, ocasionando a elevação da taxa de produção de escuma e a possível deterioração da qualidade do efluente final (LOBATO et al., 2007; SOUZA et al., 2007). Em experimento desenvolvido por Souza et al. (2006), os Sólidos em Suspensão Totais (SSTs) foram carreados da zona de digestão para a zona de decantação sem terem condições de retornar para a zona de digestão, comprometendo o teor de SSTs no efluente e na camada de escuma. 
Em uma avaliação realizada no estado do Paraná, estimou-se que o volume de escuma representa $0,12 \%$ da vazão de esgoto tratada pela Estação de Tratamento de Esgotos (ETE), predominando, entre os destinos dados após a retirada, o uso do leito de secagem, a disposição em vala dentro da própria ETE e a disposição em aterro sanitário municipal (ROSS et al., 2013).

Independentemente do processo e características de formação da escuma, há necessidade de promover seu correto descarte. Nesse sentido, este estudo busca avaliar o impacto, influência e concentração adequada da adição de escuma ao lodo de esgoto direcionado à disposição agrícola.

\section{MATERIAIS E MÉTODOS}

Este trabalho foi realizado na ETE Vassoural, em Guarapuava (PR), com coordenadas -25.383115, -51.463737 (Figura 1), que trata a vazão de 240 L/s (vazão idêntica à nominal), durante os meses de maio a agosto de 2013. Inaugurada em 2010, está localizada à margem do rio Cascavelzinho, próximo à PR170. Seu sistema de tratamento é composto pelo preliminar (gradeamento manual de $20 \mathrm{~mm}$, peneiramento de $6 \mathrm{~mm}$, desarenador ciclônico e calha Parshall), três reatores UASB com capacidade nominal de $80 \mathrm{~L} / \mathrm{s}$ e um filtro biológico percolador com distribuidor rotativo seguido de decantador para o pós-tratamento do efluente.

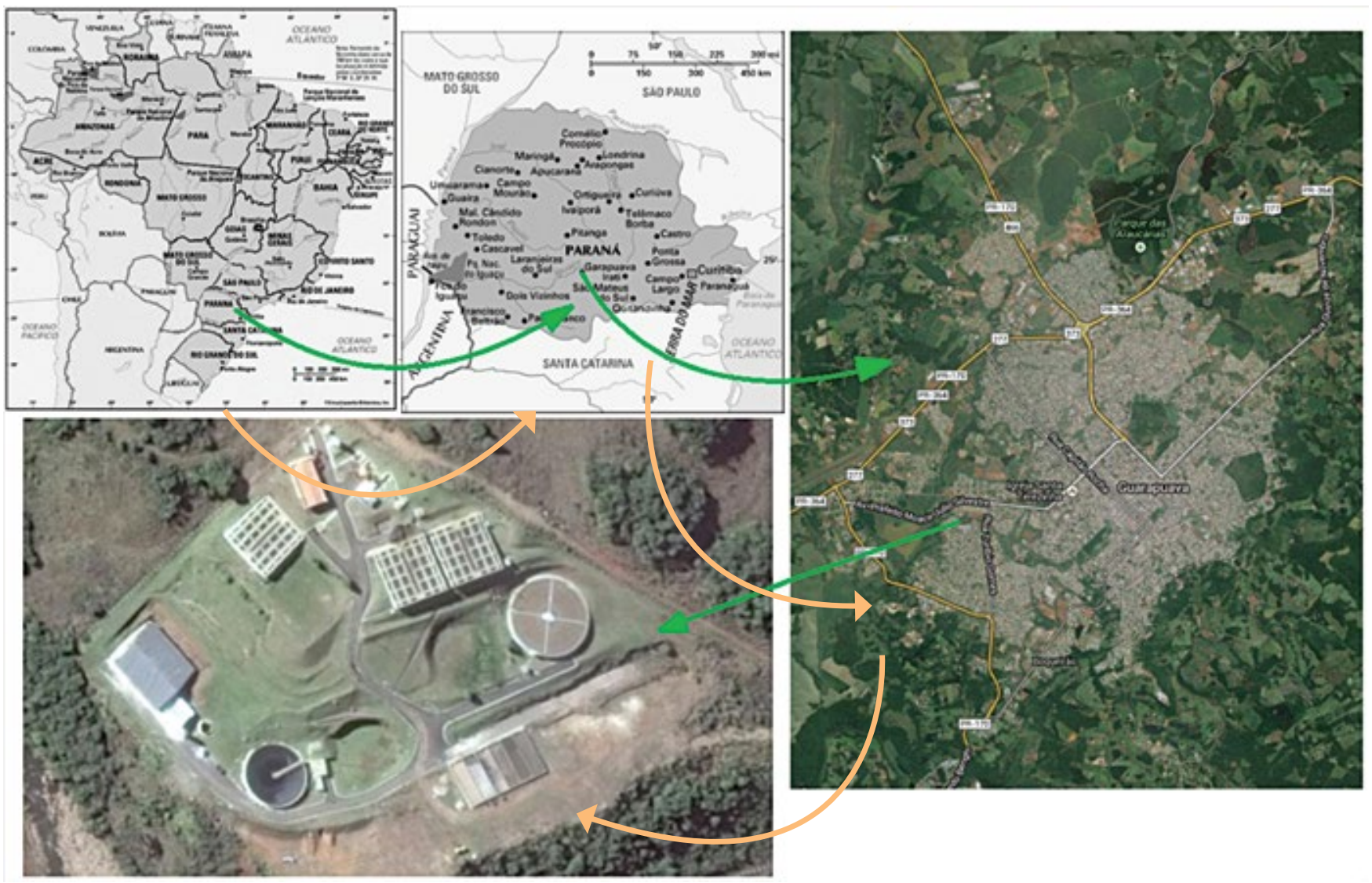

Figura 1 - Localização da ETE Vassoural - Guarapuava-PR.

Fonte: Google Maps (2015). 
A ETE realiza retiradas semanais de escuma, em um volume aproximado de $360 \mathrm{~m}^{3}$, sendo esse material desaguado em leitos de secagem, chegando ao volume de $10 \mathrm{~m}^{3}$. Os leitos de secagem foram construídos de acordo com o preconizado pela NBR 12209 (ABNT, 2011), tendo sido realizadas cinco descargas consecutivas, nos meses de junho a setembro de 2013. O leito utilizado para o desaguamento da escuma recebeu uma camada extra de areia em sua superfície; no leito utilizado para o desaguamento de lodo, a camada possuía a espessura aproximada de $2 \mathrm{~cm}$, enquanto no outro leito a espessura era de $5 \mathrm{~cm}$. Foi completada a carga máxima dos leitos, chegando a $45 \mathrm{~cm}$ de altura.

Para determinar a quantidade de escuma a ser incorporada no lodo de esgoto, considerou-se a rotina operacional de descartes de lodo e de escuma, de modo que a incorporação absorvesse todo o conteúdo de escuma descartado semanalmente na ETE. Assim, os seguintes tratamentos foram definidos:

- Tratamento 1: 10\% em massa seca de escuma desaguada em lodo de esgoto desaguado;
- Tratamento 2: $20 \%$ em massa seca de escuma desaguada em lodo de esgoto desaguado;

- Tratamento 3: 50\% em massa seca de escuma desaguada em lodo de esgoto desaguado;

- Tratamento 4: $100 \%$ de lodo (foi mantida uma amostra de lodo sem escuma para controle das alterações).

Foram avaliados os parâmetros requisitados para o lodo classe A pela Resolução CONAMA n ${ }^{\circ}$ 375/2006, que regulamenta a disposição agrícola do lodo de esgoto no Brasil (em função da ausência de uma legislação específica para escuma), complementados com a determinação da série de sólidos e O\&G. Cabe destacar que essa legislação veta a utilização de material lipídico oriundo da superfície de reatores anaeróbios na agricultura, notadamente em função do grande número de materiais inservíveis que acabam por se acumular na escuma. Apesar desse veto, torna-se importante realizar tal avaliação, para que existam novas informações e parâmetros que fomentem a revisão da legislação atual.

Quadro 1 - Parâmetros avaliados.

\begin{tabular}{|c|c|}
\hline Análise & Método \\
\hline Sólidos totais e voláteis & APHA - AWWA - WPCI - Standard Methods for the Examination of Water and Wastewater, 2012 (código 2540). \\
\hline O\&G & APHA - AWWA - WPCI - Standard Methods for the Examination of Water and Wastewater, 2012 (código 5520). \\
\hline Coliformes termotolerantes & APHA - AWWA - WPCI - Standard Methods for the Examination of Water and Wastewater, 2012 (código 9223). \\
\hline Salmonella spp. & APHA - AWWA - WPCI - Standard Methods for the Examination of Water and Wastewater, 2012 (código 9260). \\
\hline Ovos viáveis de helmintos & $\begin{array}{l}\text { US Environmental Protection Agency - Environmental Regulations and Technology-Control of Pathogens and } \\
\text { Vector Attraction in Sewage Sludge (Including Domestic Septage). Under 40 CFR Part 503. Appendix I-Test Method } \\
\text { for Detecting, Enumerating, and Determining the Viability of Ascaris Ova in Sludge, EPA/625/R-92/013, 2003, p. } 166 .\end{array}$ \\
\hline $\mathrm{N}_{\text {Kjeldahl }}, \mathrm{NH}_{3}, \mathrm{NO}_{2}^{-} \mathrm{e} \mathrm{NO}_{3}^{-}$ & APHA - AWWA - WPCI - Standard Methods for the Examination of Water and Wastewater, 2012 (código 4500). \\
\hline$P_{\text {total }}$ & APHA - AWWA - WPCI-Standard Methods for the Examination of Water and Wastewater, 2012 (código 4500P). \\
\hline $\mathrm{C}_{\text {org }}, \mathrm{K}, \mathrm{Na}, \mathrm{S}, \mathrm{Ca}$ e $\mathrm{Mg}$ & U.S. EPA - Test Methods for Evaluating Solid Waste, Physical Chemical Methods - SW-846. \\
\hline $\mathrm{Ar}, \mathrm{Ba}, \mathrm{Cd}, \mathrm{Pb}, \mathrm{Cu}, \mathrm{Cr}, \mathrm{Hg}, \mathrm{Mo}, \mathrm{Ni}, \mathrm{Se}$ e Zn & U.S. EPA - Test Methods for Evaluating Solid Waste, Physical Chemical Methods, SW-846. \\
\hline
\end{tabular}


A preservação, transporte e armazenamento das amostras para a caracterização da escuma e do lodo seguiram as especificações detalhadas pelo procedimento 9060 B - Standard Methods for the Examination of Water and Wastewater (APHA, 2012). O Quadro 1 apresenta as análises realizadas e sua respectiva metodologia, de acordo com o recomendado pela Resolução CONAMA n ${ }^{\circ}$ $375 / 2006$. As amostras foram avaliadas antes da realização das misturas e depois do término do processo de higienização.

Os parâmetros avaliados, citados no Quadro 1, foram utilizados como apoio para a comparação e verificação da possibilidade de incorporação de escuma em lodo de esgoto. Além disso, foi verificado se a mistura de escuma em lodo de esgoto atende ao preconizado na legislação brasileira (Resolução CONAMA n 375/2006), que contém os critérios e procedimentos para a disposição agrícola do lodo de esgoto, independentemente do fato de a referida legislação vetar o uso agrícola de materiais como a escuma.

A homogeneização dos tratamentos foi realizada por meio de equipamento (betoneira CSM $^{\circledR}$ 600 L), com carregador automático. Adotou-se a dosagem de $50 \%$ de cal virgem em peso seco de lodo para fins de higienização. Esse procedimento atende à legislação brasileira (CONAMA, 2006) no tocante à redução de atratividade a vetores, por permitir a permanência do $\mathrm{pH}$ das misturas acima de 12 por $2 \mathrm{~h}$ e aumentar o poder alcalinizante da mistura; consequentemente, a aceitabilidade do lodo de esgoto pelos agricultores é maior.

Para elaboração do tratamento com $100 \%$ de lodo, a betoneira foi abastecida com $250 \mathrm{~kg}$ de lodo desaguado e $125 \mathrm{~kg}$ de cal. Ligou-se o equipamento na rotação de $28 \mathrm{rpm}$, durante $10 \mathrm{~min}$, procedimento empírico para permitir a adequada mistura do lodo com a cal; na sequência, o conteúdo foi colocado em pátio coberto para aguardar o tempo de cura (30 dias). Não foi utilizada a carga completa da betoneira, para que a mistura fosse mais eficiente. 0 mesmo procedimento foi realizado para os tratamentos 1,2 e 3 .

O delineamento utilizado em campo foi de blocos ao acaso, com cinco repetições em cada, totalizando 20 parcelas amostrais. Na Figura 2, apresentam-se a escuma, o lodo e a mistura obtida no experimento, enquanto a Figura 3 mostra uma síntese do planejamento experimental. Por fim, o teste de análise de variância (ANOVA) foi aplicado para avaliação de variâncias entre os tratamentos, após a normalização dos resultados obtidos.

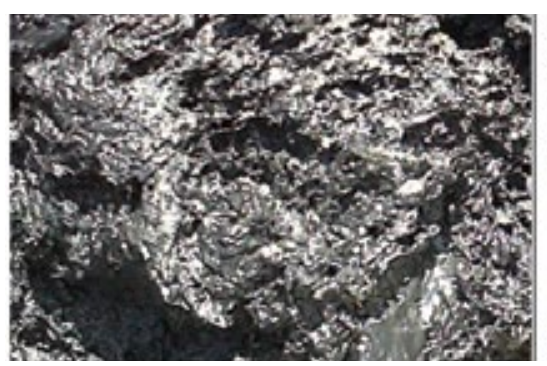

(a) Escuma desaguada

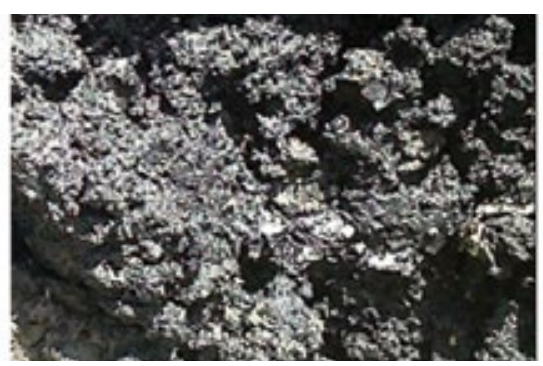

(b) Lodo de esgoto desaguado

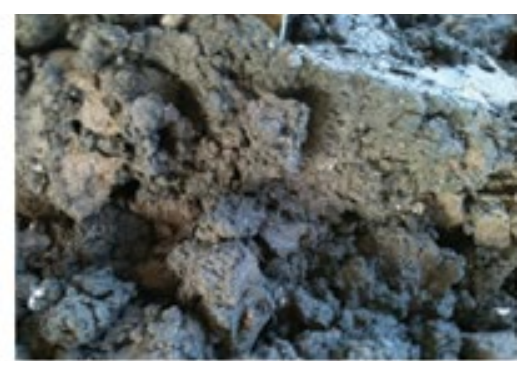

(c) Mistura pronta para estabilização

Figura 2 - Escuma e lodo desaguados antes do processo de mistura e mistura pronta para estabilização, após adição de cal virgem. 


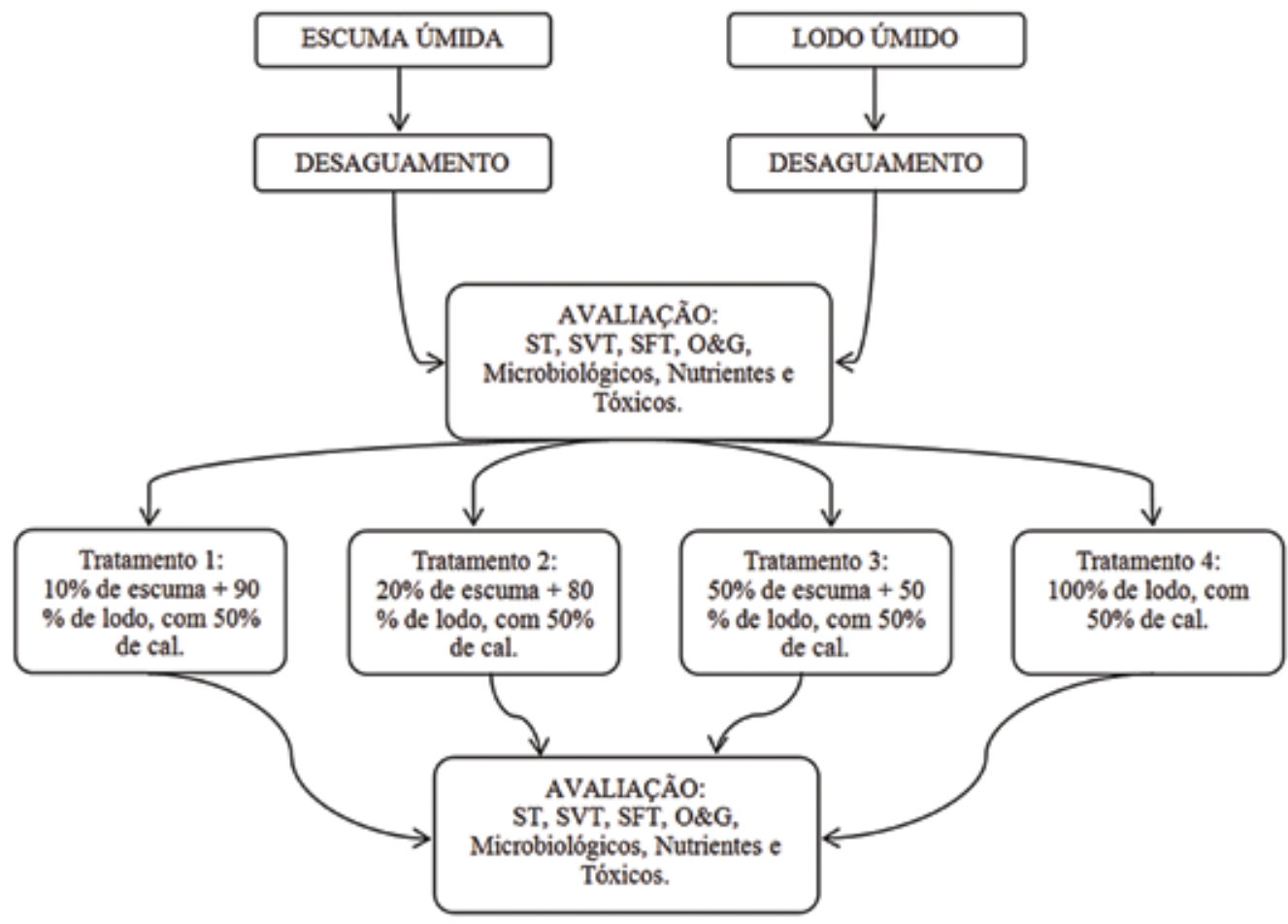

Figura 3 - Planejamento experimental para avaliar a interferência da mistura de escuma em lodo de esgoto.

\section{RESULTADOS E DISCUSSÕES}

Desaguamento de escuma e lodo de esgoto em leitos de secagem

Observa-se, na Tabela 1, que o desaguamento, tanto da escuma quanto do lodo de esgoto, ocorreu em períodos bastante curtos. $\mathrm{Na}$ descarga 1, precisou-se de um mês para atingir teores de Sólidos Totais (STs) superiores a $20 \%$, o que é justificado pelo alto índice de pluviosidade no período. $O$ experimento levou três meses para ser desenvolvido, período em que a temperatura oscilou de 5,6 a $27^{\circ} \mathrm{C}$. Apesar de ser oficialmente inverno, houve temperaturas bastante altas e com grande variação durante cada descarga. Apesar dos bons resultados no leito de secagem, o material resultante deve ser denominado "desaguado", uma vez que o teor de STs no fim do processo não ultrapassou $30 \%$. Para teores de STs superiores a $60 \%$, pode-se considerar o material "seco" (ANDREOLI et al., 2001).

Tabela 1 - Desempenho do desaguamento de escuma em função da pluviosidade no período de cada descarga.

\begin{tabular}{|c|c|c|c|c|c|c|}
\hline \multirow{2}{*}{ Descarga } & \multirow{2}{*}{$\begin{array}{l}\text { Pluviosidade total no } \\
\text { período }(\mathrm{mm})\end{array}$} & \multirow{2}{*}{ Tempo de desaguamento (dias) } & \multicolumn{2}{|c|}{ ST inicial (\%) } & \multicolumn{2}{|c|}{ ST final (\%) } \\
\hline & & & Lodo & Escuma & Lodo & Escuma \\
\hline 1 & 426,5 & 36 & 0,86 & 0,36 & 26,06 & 20,04 \\
\hline 2 & 90,0 & 13 & 6,52 & 3,50 & 19,36 & 26,61 \\
\hline 3 & 13,0 & 8 & 2,15 & 0,54 & 19,79 & 23,60 \\
\hline 4 & 0,0 & 7 & 3,72 & 0,92 & 15,69 & 17,84 \\
\hline 5 & 2,0 & 7 & 4,18 & 0,91 & 20,53 & 26,18 \\
\hline
\end{tabular}




\section{Características físico-químicas da escuma e do lodo de esgoto}

As Tabelas 2 a 4 apresentam valores de estudos de caracterização de escuma e lodo de esgoto produzidos em reatores UASB no Brasil, comparativamente aos gerados neste estudo. A escuma da ETE avaliada apresenta concentração de STs e de Sólidos Voláteis Totais (SVTs) inferior à do lodo anaeróbio, o que é esperado, pois ela não passou por nenhum processo anterior de desaguamento. $O$ tempo de acúmulo superior neste estudo e a utilização de uma escumadeira para remoção do excesso de esgoto também podem ter contribuído para essas diferenças.Quanto à relação SVT/ ST, as escumas avaliadas por Ross et al. (2013) apresentaram valores muito próximos aos de literatura, conforme apresentado na Tabela 2, sugerindo que a escuma não estava estabilizada, diferentemente do lodo de esgoto anaeróbio, que apresentou uma relação SVT/ ST de 0,47 (maior estabilidade). 0 mesmo ocorreu com a escuma da ETE Vassoural, que apresentou teor mais elevado de analitos inorgânicos comparativamente, contribuindo para essa diferenciação (Tabela 2).
O teor de O\&G da escuma apresentou significativa diferença entre as ETEs avaliadas, variando de $28 \mathrm{~g} / \mathrm{kg}$ de STs a $126 \mathrm{~g} / \mathrm{kg}$; essa variação pode ser decorrente de diferentes teores de $O \& G$ no esgoto afluente e do tempo de acúmulo da escuma. A escuma da ETE Vassoural não foi avaliada quanto ao $\mathrm{pH}$, Demanda Química de Oxigênio (DQO) e Demanda Bioquímica de Oxigênio (DBO) (Tabela 1).

Os valores encontrados e esperados para coliformes termotolerantes e Salmonella spp. (Tabela 3) no lodo e escuma foram elevados quando comparados aos limites preconizados pela Resolução CONAMA n³75/2006, visto que os materiais não eram higienizados, evidenciando a necessidade de controle microbiológico.

Os valores de $\mathrm{P}_{\text {total }}$ na escuma e lodo da ETE Vassoural foram muito próximos; o mesmo aconteceu para $\mathrm{N}_{\text {Kjechal }}$ e Ca, sendo os valores da ETE Vassoural menores que os observados por Ross et al. (2013) em trabalho sobre a região da Grande Curitiba. Ainda, para a série nitrogenada, $\mathrm{S}, \mathrm{K}$ e Na, a escuma da ETE Vassoural apresentou teores inferiores aos observados no estudo citado. Já no lodo, os teores de $\mathrm{S}$ e K foram menores que na escuma, enquanto os de $\mathrm{Na}$ e $\mathrm{Mg}$ foram maiores (Tabela 4).

Tabela 2 - Teores observados na escuma e lodo de esgoto da ETE Vassoural e outros trabalhos correlatos.

\begin{tabular}{|c|c|c|c|c|c|c|c|c|}
\hline \multirow{3}{*}{$\begin{array}{c}\text { Parâmetro } \\
\text { (unidade) }\end{array}$} & \multicolumn{6}{|c|}{ Escuma } & \multicolumn{2}{|c|}{ Lodo } \\
\hline & \multirow{2}{*}{ ETE Vassoural 1} & \multicolumn{2}{|c|}{ Ross et al. (2013) } & \multirow{2}{*}{$\begin{array}{l}\text { Souza et al. } \\
\text { (2007) }\end{array}$} & \multirow{2}{*}{$\begin{array}{l}\text { Oliveira et al. } \\
\text { (2007) }\end{array}$} & \multirow{2}{*}{$\begin{array}{l}\text { Souza et al. } \\
\text { (2006) }\end{array}$} & \multirow{2}{*}{ ETE Vassoural ${ }^{1}$} & \multirow{2}{*}{$\begin{array}{l}\text { Bittencourt } \\
\text { et al. (2011, } \\
2012)^{2}\end{array}$} \\
\hline & & ETE Atuba Sul & ETE Padilha Sul & & & & & \\
\hline STs (\%) & $22,87( \pm 3,85)$ & $18,18( \pm 4,94)$ & $18,75( \pm 3,95)$ & 11,2 & 3,8 & 9 & $20,29( \pm 3,73)$ & 51,7 \\
\hline SVTs (\%) & $12,08( \pm 4,65)$ & $13,01( \pm 4,57)$ & $11,86( \pm 1,74)$ & NR & NR & NR & $12,02( \pm 4,58)$ & 23,23 \\
\hline SVT/ST (---) & 0,53 & 0,71 & 0,64 & 0,74 & 0,73 & 0,7 & 0,59 & 0,47 \\
\hline$O \& G(\mathrm{~g} / \mathrm{kg})$ & $126( \pm 81)$ & $66( \pm 29)$ & $28( \pm 15)$ & NR & NR & NR & $91( \pm 98)$ & NR \\
\hline
\end{tabular}

Notas: ${ }^{1}$ DP = desvio padrão, avaliação de cinco amostras. ${ }^{2}$ Lodo submetido ao processo de estabilização alcalina prolongada. NR = não realizado. Os resultados de avaliação deste trabalho e os obtidos por ROSS et al. (2013) decorrem de um estudo em escala real, enquanto os apresentados por Souza et al. (2006), Souza et al. (2007) e Oliveira et al. (2007) ocorreram em estação-piloto; nestes casos, o controle de qualidade sobre o esgoto bruto é muito maior, assim como as variações são menores. 
Tabela 3 - Teores de micro-organismos observados na escuma e lodo de esgoto da ETE Vassoural e outros trabalhos correlatos.

\begin{tabular}{|c|c|c|c|c|c|}
\hline \multirow{3}{*}{$\begin{array}{l}\text { Parâmetro } \\
\text { (unidade) }\end{array}$} & \multicolumn{3}{|c|}{ Escuma } & \multirow{3}{*}{$\begin{array}{l}\text { Limite } \\
\text { Resolução CONAMA no } \\
375 / 2006\end{array}$} & \multirow{3}{*}{$\begin{array}{c}\text { Lodo } \\
\text { ETE Vassoural }^{1}\end{array}$} \\
\hline & \multirow{2}{*}{ ETE Vassoural $^{1}$} & \multicolumn{2}{|c|}{ Ross et al. (2013) } & & \\
\hline & & ETE Atuba Sul & ETE Padilha Sul & & \\
\hline $\begin{array}{c}\text { Coliformes } \\
\text { termotolerantes } \\
\text { (UFC/g) }\end{array}$ & $24206( \pm 48081)$ & $22337( \pm 17556)$ & $38698( \pm 25655)$ & $<1.000$ & $15252( \pm 26367)$ \\
\hline Salmonella spp. (UFC/g) & $19374( \pm 27475)$ & $103015( \pm 18473)$ & $3844( \pm 3005)$ & $0 / 10 \mathrm{~g}$ de ST & $15015( \pm 19709)$ \\
\hline $\begin{array}{l}\text { Ovos viáveis de } \\
\text { helmintos (Ovo/g) }\end{array}$ & $1,40( \pm 0,55)$ & NR & NR & $<0,25$ & $<1( \pm 0,00)$ \\
\hline
\end{tabular}

Notas: ${ }^{1}$ DP = desvio padrão, avaliação de cinco amostras. UFC = unidade formadora de colônia. NR = não realizado.

Tabela 4 - Teores de elementos químicos observados na escuma e lodo de esgoto da ETE Vassoural e outros trabalhos correlatos.

\begin{tabular}{|c|c|c|c|c|c|c|c|}
\hline \multirow{3}{*}{$\begin{array}{l}\text { Parâmetro } \\
\text { (unidade) }\end{array}$} & \multirow{3}{*}{$\begin{array}{l}\text { ETE Vassoural } 1 \\
\text { ETE Atuba Sul }\end{array}$} & \multicolumn{2}{|l|}{ scuma } & \multirow{3}{*}{$\begin{array}{l}\text { Limite } \\
\text { CONAMA }\end{array}$} & \multicolumn{3}{|c|}{ Lodo } \\
\hline & & \multicolumn{2}{|c|}{ Ross et al. (2013) } & & \multirow{2}{*}{ ETE Vassoural ${ }^{1}$} & \multirow{2}{*}{$\begin{array}{c}\text { Bittencourt et al. } \\
(2011,2012)^{2}\end{array}$} & \multirow{2}{*}{ 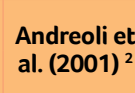 } \\
\hline & & ETE Padilha Sul & ETE Padilha Sul & & & & \\
\hline $\mathrm{C}_{\text {org }}(\%)$ & $24,26( \pm 10,37)$ & $52,01( \pm 26,00)$ & $65,48( \pm 16,24)$ & NE & $15,52( \pm 13,60)$ & $N R$ & 20,1 \\
\hline $\mathrm{P}_{\text {total }}(\%)$ & $0,087( \pm 0,10)$ & $2,97( \pm 3,83)$ & $1,02( \pm 0,95)$ & NE & $0,111( \pm 0,14)$ & NR & 0,67 \\
\hline $\mathrm{N}_{\text {Kjedhal }}(\%)$ & $0,33( \pm 0,29)$ & $1,24( \pm 0,44)$ & $1,54( \pm 0,32)$ & NE & $0,32( \pm 0,27)$ & NR & 2,22 \\
\hline $\mathrm{N} \mathrm{NH}_{4}^{+}(\mathrm{mg} / \mathrm{kg})$ & $40,64( \pm 19,38)$ & $2828( \pm 2527)$ & $2695( \pm 1709)$ & NE & $27,22( \pm 14,77)$ & NR & NR \\
\hline $\mathrm{N} \mathrm{NO}_{3}^{-}(\mathrm{mg} / \mathrm{kg})$ & $62,94( \pm 137,67)$ & $63,53( \pm 18,99)$ & $74,70( \pm 36,85)$ & NE & $2,86( \pm 4,59)$ & NR & NR \\
\hline $\mathrm{NNO}_{2}^{-}(\mathrm{mg} / \mathrm{kg})$ & $4,92( \pm 7,65)$ & $16,92( \pm 12,86)$ & $20,36( \pm 6,37)$ & $\mathrm{NE}$ & $<1,0( \pm 0)$ & NR & NR \\
\hline K(\%) & $0,021( \pm 0,02)$ & $0,24( \pm 0,11)$ & $0,60( \pm 0,43)$ & NE & $0,037( \pm 0,04)$ & NR & 0,95 \\
\hline $\mathrm{Na}(\%)$ & $0,028( \pm 0,02)$ & $0,81( \pm 0,38)$ & $1,02( \pm 0,56)$ & NE & $0,047( \pm 0,04)$ & NR & NR \\
\hline $\mathrm{S}(\mathrm{mg} / \mathrm{kg})$ & $8,88( \pm 14,97)$ & $18,19( \pm 21,52)$ & $16,61( \pm 8,77)$ & NE & $3,29( \pm 4,43)$ & NR & NR \\
\hline $\mathrm{Ca}(\%)$ & $0,925( \pm 0,38)$ & $27,06( \pm 14,22)$ & $21,39( \pm 9,34)$ & NE & $0,803( \pm 0,43)$ & NR & 0,83 \\
\hline Mg (\%) & $0,265( \pm 0,23)$ & $5,14( \pm 3,24)$ & $4,78( \pm 2,99)$ & $\mathrm{NE}$ & $0,359( \pm 0,35)$ & NR & 0,3 \\
\hline As (mg/kg) & $<10( \pm 0)$ & $<10( \pm 0)$ & $<10( \pm 0)$ & 41 & $<10( \pm 0)$ & 7,9 & NR \\
\hline $\mathrm{Ba}(\mathrm{mg} / \mathrm{kg})$ & $<10( \pm 0)$ & $<10( \pm 297)$ & $<10( \pm 0)$ & 1300 & $<10( \pm 0)$ & 106 & $N R$ \\
\hline $\mathrm{Cd}(\mathrm{mg} / \mathrm{kg})$ & $2,4( \pm 3,0)$ & $<1( \pm 0)$ & $<1( \pm 0)$ & 39 & $1,8( \pm 1,7)$ & 5,2 & NR \\
\hline $\mathrm{Pb}(\mathrm{mg} / \mathrm{kg})$ & $19( \pm 12)$ & $<1( \pm 18)$ & $<1( \pm 0)$ & 300 & $18( \pm 23)$ & 44 & NR \\
\hline $\mathrm{Cu}(\mathrm{mg} / \mathrm{kg})$ & $66( \pm 19)$ & $70( \pm 57)$ & $79( \pm 54)$ & 1500 & $88( \pm 62)$ & 99 & NR \\
\hline $\mathrm{Cr}(\mathrm{mg} / \mathrm{kg})$ & $10( \pm 11)$ & $<1( \pm 5)$ & $<1( \pm 0)$ & 1000 & $14( \pm 19)$ & $<2,0$ & NR \\
\hline $\mathrm{Hg}(\mathrm{mg} / \mathrm{kg})$ & $<10( \pm 0)$ & $<10( \pm 0)$ & $<10( \pm 0)$ & 17 & $<10( \pm 0)$ & 0,4 & NR \\
\hline Mo (mg/kg) & $<10( \pm 0)$ & $<10( \pm 0)$ & $<10( \pm 0)$ & 50 & $<10( \pm 0)$ & 13 & $N R$ \\
\hline $\mathrm{Ni}(\mathrm{mg} / \mathrm{kg})$ & $40( \pm 75)$ & $<1( \pm 0)$ & $<1( \pm 0)$ & 420 & $14( \pm 19)$ & 20 & NR \\
\hline $\mathrm{Se}(\mathrm{mg} / \mathrm{kg})$ & $<1( \pm 0)$ & $<10( \pm 0)$ & $<10( \pm 0)$ & 100 & $<1( \pm 0)$ & 1,1 & NR \\
\hline $\mathrm{Zn}(\mathrm{mg} / \mathrm{kg})$ & $126( \pm 106)$ & $227( \pm 97)$ & $474( \pm 491)$ & 2800 & $316( \pm 322)$ & 916 & $N R$ \\
\hline
\end{tabular}

Notas: ${ }^{1}$ DP = desvio padrão, avaliação de cinco amostras. ${ }^{2}$ lodo submetido ao processo de estabilização alcalina prolongada. NE = não existente. NR = não realizado. 
As espécies inorgânicas encontradas na escuma foram comparadas com o lodo de ETE avaliado por Bittencourt et al. (2012). Os elementos Ba, Cd, $\mathrm{Pb}$, Cu e $\mathrm{Zn}$ apresentaram-se em valores inferiores aos encontrados em lodo de esgoto, enquanto As, $\mathrm{Hg}, \mathrm{Mo} \mathrm{Ni}$ e Se foram semelhantes e $\mathrm{Cr}$ e Ni, ligeiramente superiores. Cabe ressaltar que em todos os casos os valores foram bem inferiores aos preconizados na legislação (Tabela 4). Por outro lado, os teores de $\mathrm{Cd}, \mathrm{Pb}, \mathrm{Cu}, \mathrm{Cr}$ e $\mathrm{Ni}$ foram expressivos, quando comparados ao encontrado no trabalho de Ross et al. (2013). Acredita-se que pode ser devido ao uso de agrotóxicos na bacia, os quais chegam à ETE por meio de runoff, infiltração na rede ou mesmo devido à menor diluição de cargas tóxicas (Tabela 4).

\section{AVALIAÇÃO DAS MISTURAS DE ESCUMA E LODO DE ESGOTO}

Os parâmetros dos tratamentos avaliados estão apresentados nas Figuras 4 a 8 . Os dados foram avaliados quanto à influência dos componentes da escuma e do lodo de esgoto nos tratamentos e suas tendências de comportamento.
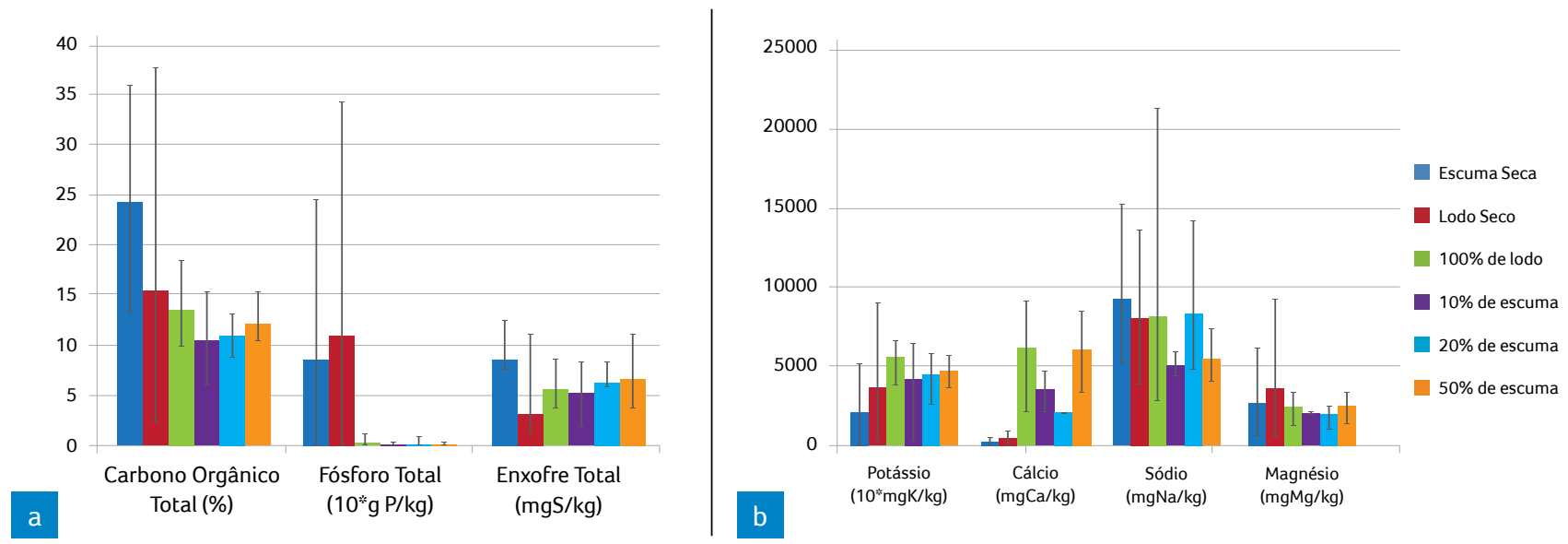

Nota: Valores de P e Kampliados em escala de dez vezes.

Figura 4 - Influência da adição de escuma em lodo de esgoto para (a) $\mathrm{C}_{\text {org }}, \mathrm{P}_{\mathrm{t}}$ e S e (b) $\mathrm{K}, \mathrm{Ca}$, Na e Mg.

O teor de $\mathrm{C}_{\text {org }}$ (Figura 4a) no lodo de esgoto desaguado e na escuma foi superior aos teores encontrados nas amostras com $100 \%$ de lodo e nas misturas. Assim, após as misturas, observou-se uma redução desses valores, devido provavelmente à adição de lodo e do efeito da adição da cal. O $P_{\mathrm{t}}$ (Figura 4a) apresentou significativa redução nos materiais desaguados; antes da higienização, estes já possuíam teores superiores aos encontrados após o procedimento de caleação. Andreoli (1999) já observa- va a influência da calagem na redução dos teores de $P_{t}$ no lodo para uso agrícola. Por sua vez, para S (Figura 4a), Na, K e Mg (Figura 4b), entende-se que não houve variação expressiva em suas concentrações, não havendo interferência nem da adição de escuma nas misturas, nem da cal para a higienização. Já os teores de Ca das misturas (Figura 4b) foram bem superiores aos encontrados antes do procedimento de higienização, fato já esperado, uma vez que o agente sanitizante é à base de $\mathrm{CaO}$. 


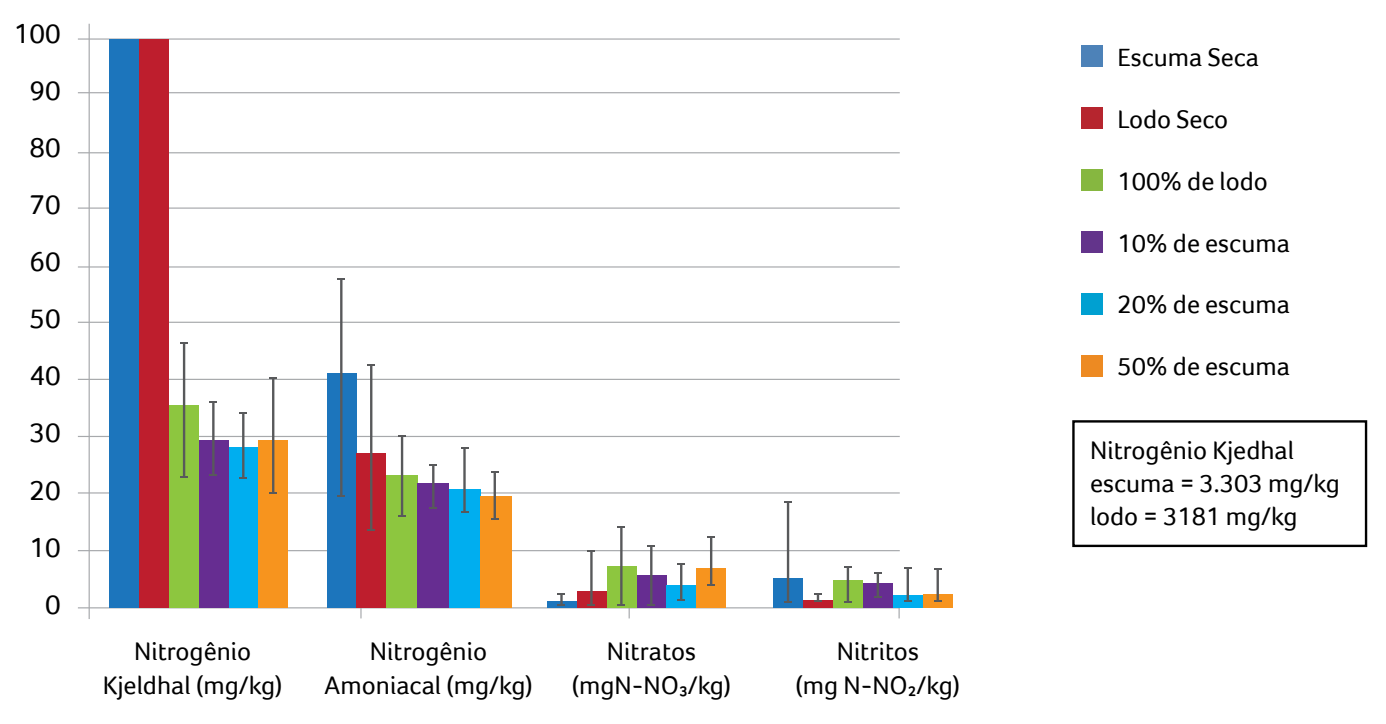

Figura 5 - Comportamento de $\mathrm{N}$ em relação à adição de escuma em lodo de esgoto.

Para N, observa-se na Figura 5 que a elevação do $\mathrm{pH}$ ocasionada pela adição de $\mathrm{CaO}$ proporcionou uma elevada liberação de gases nitrogenados; já os teores de $\mathrm{N}_{\text {Kjechal }}$ reduziram bastante nas misturas e nas amostras com $100 \%$ de lodo. N é o componente de principal interesse no lodo de esgoto, muitas vezes utilizado como fator limitante para a definição da dosagem máxima de aplicação em solos, devido à possibilidade de lixiviação em forma de nitratos e, consequentemente, contaminação do freático (MALAVOLTA, 1980; MELLO, 1984). Entre as formas de $\mathrm{N}, \mathrm{NH}_{4}^{+}, \mathrm{NO}_{3}^{-}$e $\mathrm{NO}_{2}^{-}$são encontrados em menor quantidade. A volatilização de $\mathrm{NH}_{4}^{+}$, a instabilidade de $\mathrm{NO}_{2}{ }^{-}$e o potencial de lixiviação de $\mathrm{NO}_{3}{ }^{-}$são fatores que podem estar associados a isso.

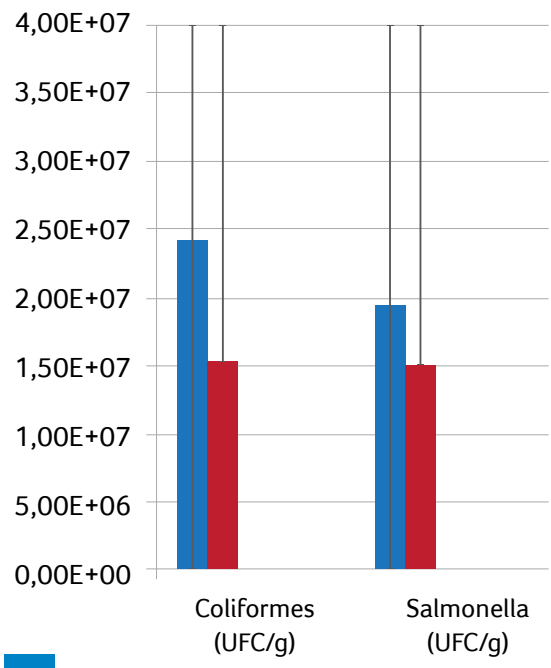

a

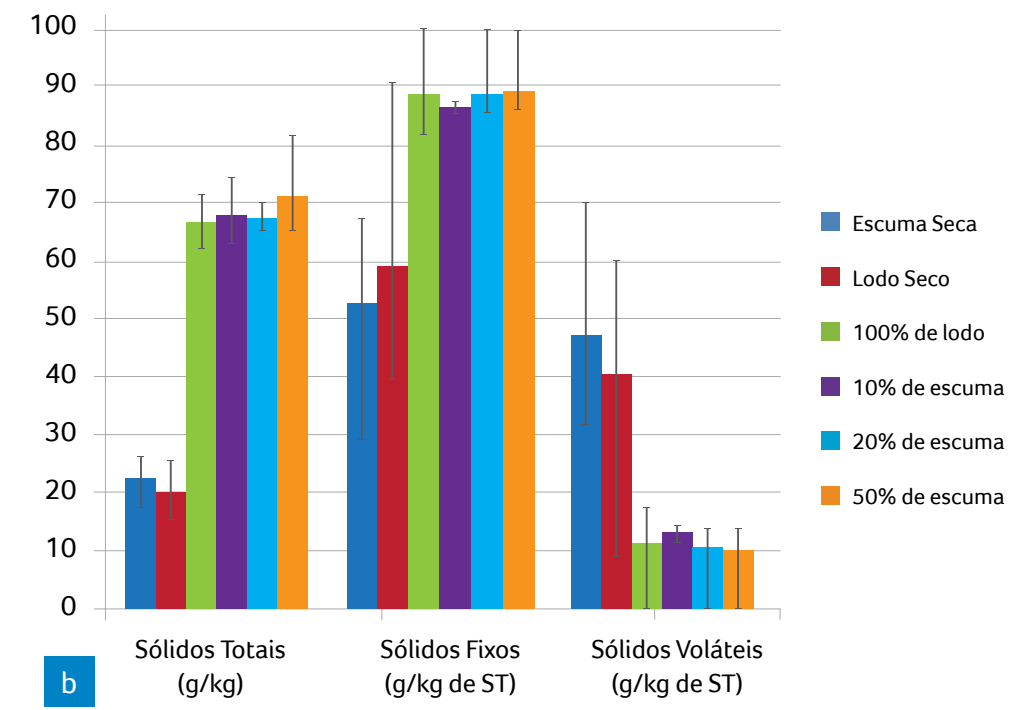

Figura 6 - Impacto da adição de escuma em lodo de esgoto para (a) coliformes termotolerantes, ovos viáveis de helmintos e Salmonella spp. e (b) STs, sólidos fixos e voláteis. 
Em relação à atividade microbiológica (Figura $6 a)$, verifica-se que o processo de estabilização alcalina prolongada foi bastante efetivo; anteriormente, encontravam-se quantidades significativas de coliformes termotolerantes e Salmonela spp., praticamente eliminadas com a adição de $\mathrm{CaO}$. Já os valores para contagem de ovos viáveis de helmintos, tanto antes da higienização quanto depois desse procedimento, indicaram média para todas as amostras de $<1$ ovo/g de STs para o lodo e 1,4 ovo/g de STs para a escuma; por essa razão, não foram exibidos em figuras.

A higienização de lodos pela via química com uso de $\mathrm{CaO}$ elimina os micro-organismos pela alteração da natureza coloidal do protoplasma celular e também resulta em elevação do $\mathrm{pH}$ da massa de lodo, maximizando a efetividade do processo. O lodo será considerado seguro caso o $\mathrm{pH}$ tenha ficado acima de 12 por no mínimo $72 \mathrm{~h}$, a temperatura seja superior a $52{ }^{\circ} \mathrm{C}$ por 12 h, minimamente, e seque ao ambiente até que o teor de sólidos seja superior a 50\% (ANDREO$\mathrm{LI}$ et al., 2001). Observa-se na Figura 6b que as amostras após a caleação tiveram o teor de STs acima de $50 \%$ em todos os casos, evidenciando que houve higienização. A caleação também contribuiu para o aumento no teor de sólidos fixos das misturas.

Os elementos inorgânicos avaliados ( $\mathrm{Cu}, \mathrm{Zn}$ e $\mathrm{Cr}$ ) são micronutrientes necessários às plantas, mas podem assumir condição fitotóxica se em altas concentrações, muito embora esses limiares variem significativamente entre os vegetais (MARSCHNER, 1986; MENGEL; KIRKBY, 1987). Os elementos $\mathrm{Pb}$ e $\mathrm{Cr}$ podem estar presentes no $\mathrm{CaO}$ e isso pode explicar o pequeno aumento observado nas misturas. Já $\mathrm{Ni}, \mathrm{Zn}$ e Cd não apresentaram alteração entre os tratamentos e os materiais brutos. Observou-se que o lodo e a escuma desaguados possuíam teores muito superiores de Cu antes da aplicação da cal. Ressalta-se que a análise foi realizada por absorção atômica, com abertura da amostra com digestão ácida com ácido nítrico e clorídrico, assim como todos os elementos do grupo inorgânico, o que corresponde à determinação do valor total do elemento por $\mathrm{kg}$ de amostra seca. Acredita-se que o elemento possa ter complexado e se depositado no fundo do local de armazenagem, ficando excluído da amostragem.

Os elementos $\mathrm{Ba}, \mathrm{As}, \mathrm{Hg}$, Mo e Se não apresentaram variação durante as análises, estando bem abaixo dos limites estipulados por lei.
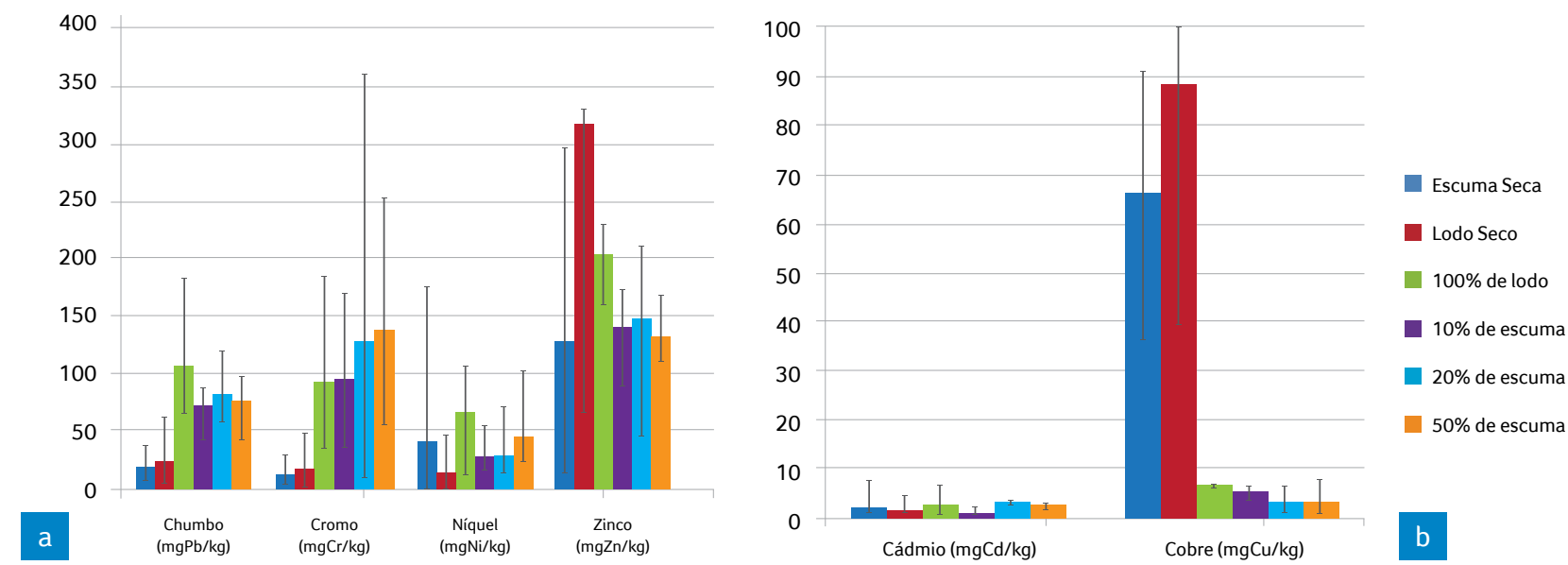

Figura 7 - Impacto da adição de escuma em lodo de esgoto para (a) Pb, Cr, Ni e Zn e (b) Cd e Cu. 


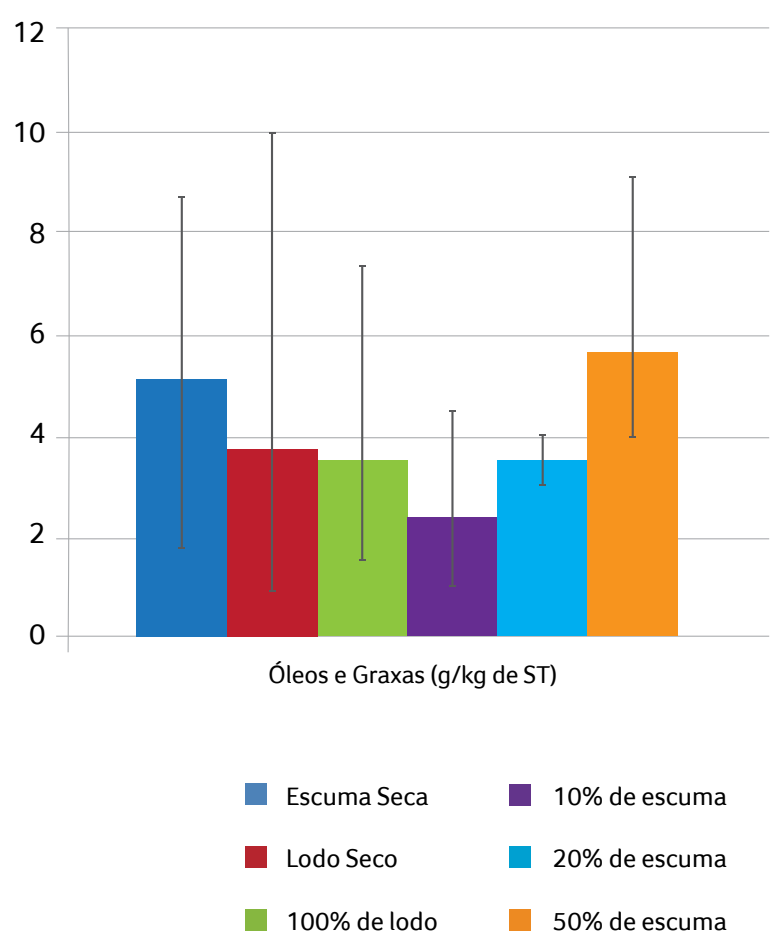

Figura 8 - Comportamento do teor de $O \& G$ em relação à adição de escuma em lodo de esgoto.

A incorporação de escuma no lodo de esgoto tende a causar um aumento no teor de O\&G (Figura 8), porém a mistura com $20 \%$ de escuma apresentou um resultado ligeiramente inferior, diferindo do esperado.

Não foram observadas variâncias significativas $\left(P_{0,05}\right)$ entre as médias, o que indica que a inclusão de escuma nas proporções de $10 \%, 20 \%$ e $50 \%$ não alterou estatisticamente a composição do lodo de esgoto para nenhuma das variáveis, mesmo com o grande desvio padrão existente entre os resultados, que foi minimizado pela normalização dos dados. Os parâmetros de Salmonella spp. e as espécies inorgânicas As, $\mathrm{Ba}, \mathrm{Hg}$, Mo e Se não variaram durante as análises; por essa razão, não foram submetidos à avaliação estatística. A fim de proceder a uma avaliação estatística mais consistente, seriam necessárias mais amostragens, que permitiriam uma redução no desvio padrão encontrado.

\section{CONCLUSÕES}

Foram desaguados separadamente em leitos de secagem cinco lotes de escuma e cinco lotes de lodo anaeróbio de esgoto e, após esse procedimento, foram realizadas três misturas com as dosagens de $10 \%, 20 \%$ e $50 \%$ de escuma em lodo e um lote com $100 \%$ de lodo foi mantido. Os tratamentos passaram pelo processo de estabilização alcalina prolongada pela adição de cal em $50 \%$ do peso seco, os lotes foram avaliados em laboratório e os resultados receberam tratamento estatístico.

Os teores de $\mathrm{C}_{\text {org }}$ e de $\mathrm{P}_{\text {total }}$ apresentaram redução, ou seja, os materiais desaguados, antes da mistura e higienização, possuíam teores superiores ao encontrado após o procedimento de caleação. Os teores de Ca das misturas foram bem superiores aos encontrados antes do procedimento de higienização, fato já esperado, visto que o agente sanitizante é a cal. Com relação aos elementos $\mathrm{S}, \mathrm{Na}, \mathrm{Ke} \mathrm{Mg}$, entende-se que não houve variação significativa em suas concentrações, não havendo interferência nem da adição de escuma nas misturas, nem da cal para a higienização. Quanto à avaliação do conjunto de N, tem-se que a elevação do $\mathrm{pH}$, ocasionada pela adição de cal, proporcionou uma liberação de $\mathrm{N}$ para a atmosfera. Encontraram-se quantidades significativas de coliformes termotolerantes e Salmonela spp. nos lotes de escuma e lodo desaguados, porém com o processo de higienização as concentrações desses micro-organismos ficaram bem abaixo do exigido pela Resolução CONOMA n 375/2006. Com relação à contagem de ovos de helmintos, tanto antes da higienização das misturas quanto depois desse procedimento, obteve-se a média de 1 ovo/g de matéria seca. Não foi observada diferença significativa entre os valores encontrados para os elementos inorgânicos avaliados e o teor de sólidos foi aumentado para todos os tratamentos. A incorporação de escuma no lodo de esgoto tende a causar um aumento no teor de $O \& G$, exceto na mistura com $20 \%$ de escuma, que apresentou um resultado ligeiramente inferior, diferindo do esperado. 
As amostras foram submetidas à avaliação estatística pelo teste ANOVA, o qual não encontrou diferenciação estatística entre as médias, significando que a inclusão de escuma nas proporções de 10\%, 20\% e $50 \%$ não alterou a composição do lodo de esgoto destinado ao uso agrícola.

\section{REFERÊNCIAS}

ASSOCIAÇÃO BRASILEIRA DE NORMAS TÉCNICAS. ABNT, NBR 12.209: Elaboração de Projetos Hidráulicos-Sanitários de Estações de Tratamento de Esgotos Sanitários. Rio de Janeiro, 2011.

ANDREOLI, C. V.. Uso e manejo do lodo de esgoto na agricultura e sua influencia em características ambientais no agroecossistema. 1999. 278 p. Tese (Doutorado em Meio Ambiente e Desenvolvimento) Universidade Federal do Paraná, 1999.

ANDREOLI, C. V.; VON SPERLING, M.; FERNANDES, F.. (Org.) Lodo de esgotos: tratamento e disposição final. Belo Horizonte: Departamento de Engenharia Sanitária e Ambiental, UFMG; Curitiba: SANEPAR, 2001. 484 p. (Princípios do tratamento biológico de águas residuárias, v. 6).

APHA, AWWA, WEF. Standard methods for examination of water and wastewater, 22. ed. Washington: Publication Office American Public Health Association, APHA, AWWA, WEF, 2012.

BITTENCOURT, S.; SERRAT, B.M.; AISSE, M.M.; GOMES, D.. Sewage sludge usage in agriculture: a case study of its destination in $\mathrm{Cu}$ ritiba Metropolitan Region, Paraná, Brazil. In: SIMPÓSIO LATINO AMERICANO DE DIGESTÃO ANAERÓBIA, 10, Minas Gerais, 23-27 out. 2011. 1CD-ROM.

BITTENCOURT, S.; SERRAT, B. M.; AISSE, M.M.; MARIN, L.M.K.S.; SIMÃO, C.C.. Aplicação de lodos de estações de tratamento de água e de tratamento de esgoto em solo degradado. Engenharia Sanitária e Ambiental, v. 17, p. 315-324, 2012.

BRASIL. Conselho Nacional de Meio Ambiente, CONAMA. Resolução n. 375, de 29 de agosto de 2006. Define critérios e procedimentos, para o uso agrícola de lodos de esgoto gerados em estações de tratamento de esgoto sanitário e seus produtos derivados, e dá outras providências.

CHERNICHARO, C.A.L.. Tecnologias Nacionais para Tratamento Anaeróbio de Esgotos. Seminário sobre Gerenciamento do Saneamento em Comunidades Planejadas. Curitiba: Alphaville Graciosa, 2004.

LOBATO, L.C.S.; CHERNICHARO, C.A.L.; OLIVEIRA FILHO, J.M.; MORAES, O.J.S.. Avaliação de desempenho da pré-operação dos re- atores UASB ETE Onça: capacidade instalada 2,05 m³/s. In: CONGRESSO BRASILEIRO DE ENGENHARIASANITÁRIA E AMBIENTAL, 24, Belo Horizonte, 3-7 set. ABES, 2007. 1CD ROM.

MALAVOLTA, E. (1980) Manual de química agrícola: nutrição de plantas e fertilidade do solo. São Paulo, Edit. Agronômica Ceres, $528 \mathrm{p}$.

MARSCHNER, H. (1986). Mineral Nutrition of Higher Plants. London, Academic Press, 674 p.

MELLO, F.A.F.; BRASIL SOBRINHO, M.O.C.; ARZOLLA, S.; SILVEIRA, R.I.; NETTO A.C.; KIEHL J.C.. Fertilidade do solo. 2 ed. Piracicaba, SP, 1984. p.400.

MENGEL, K.; KIRKBY, E.A. (1987). Principles of Plant Nutrition. $4^{\mathrm{a}}$ edição, Editora International Potash Institute, Switzerland, 665 p.

OLIVEIRA, J.P.; SILVA, J.S.C.; PEREIRA NETO, G.P.; BASTOS, G.S.M.; CHERNICHARO, C.A.L.. Avaliação comparativa do desempenho de reator UASB convencional e reator UASB com dois estágios de coleta de biogás no tratamento de esgotos domésticos. In: CONGRESSO BRASILEIRO DE ENGENHARIA SANITÁRIA E AMBIENTAL, 24, Belo Horizonte, 3-7 set. ABES, 2007. 1CD-ROM.

ROSS, B.Z.L.; CARNEIRO, C.; AISSE, M.M.; FROEHNER S.. Caracterização da escuma produzida em reatores anaeróbios de manta de lodo, estado do Paraná. In: CONGRESSO BRASILEIRO DE ENGENHARIA SANITÁRIA E AMBIENTAL, 27, 2013, Goiânia. Anais... Goiânia: ABES, 2013. 1 pen drive.

SILVA, S.Q.; ZERBINI, A.M.; GODINHO, V.M.; CHERNICHARO, C.A.L. (2005) Caracterização morfológica de micro-organismos presentes em escuma de reatores UASB tratando esgotos domésticos. In: CONGRESSO BRASILEIRO DE ENGENHARIA SANITÁRIA E AMBIENTAL, 23, Campo Grande 18-23 set. ABES, 2005. 1CD-ROM.

SOUZA, C.L.. Estudo quantitativo e qualitativo de escuma acumulada em reatores UASB tratando esgotos domésticos. $105 \mathrm{f}$. Dissertação (Mestrado em Saneamento, Meio Ambiente e Recursos Hídricos), Universidade Federal de Minas Gerais, Belo Horizonte, 2006.

SOUZA, C.L.; AQUINO, S.F.; CHERNICHARO, C.A.L.. Production and characterization of scum and its role in odour control in UASB reactors treating domestic wastewater. Water Science \& Technology, v. 54, p. 201-208, 2006.

WEF (WATER ENVIRONMENT FEDERATION); AMERICAN SOCIETY OF CIVIL ENGINEERS (ASCE); ENVIRONMENTAL AND WATER RESOURCES INSTITUTE (EWRI). Biological nutrient removal operation in wastewater treatment plant. Manual of practice 29. WEF, ASCE, EWRI, USA, 2005. 